\title{
Naturopathic Treatment of Obesity
}

\author{
Mary Koithan, PhD, RN-C, CNS-BC ${ }^{1,3}$ and Elizabeth Sutherland, ND ${ }^{2,3}$ \\ 1 University of Arizona, College of Nursing \\ 2 National College of Natural Medicine \\ 3 University of Arizona, Department of Family and Community Medicine
}

\section{Holistic Approaches to Obesity}

Because of the complex nature of obesity, a holistic approach is often identified as best practice. Whole systems of complementary medicine (WSCAM), "complete systems of theory and practice that have evolved independently from or parallel to allopathic (conventional) medicine", can offer this type of an approach. ${ }^{1}$ WSCAM, including Traditional Chinese Medicine, Ayurveda, Naturopathy and classical homeopathy, are traditional historical systems of medicine that are practiced by individual cultures. These WSCAM share a perspective that imbalances in the patient's overall constitution are at the root of disease and dysfunction, including obesity. Therefore, treatment focused on the person's overall well-being would ultimately address obesity. A particular approach that is frequently used to treat obesity is Naturopathic Medicine.

\section{What Is Naturopathic Medicine?}

Although the roots of Naturopathy trace back through Europe, modern Naturopathic Medicine was formalized in 1896 by Benedict Lust, an American physician. Naturopathic doctors (NDs) are graduates of accredited, four-year post-baccalaureate programs, trained in basic and clinical sciences as well as in a wide range of complementary treatment modalities. Regulation of ND practice varies widely in the US. Therefore, if referring to an ND for the purpose of holistic weight loss support, the nurse practitioner would be wise to recommend an ND certified by the North American Board of Naturopathic Examiners (NABNE) and graduated from a program recognized by the Council on Naturopathic Medicine (CNME). A list of qualified naturopathic physicians can be found at http://heartspring.net/naturopathic_directory.html.

NDs are trained as primary care providers and are recognized for clinical expertise and effectiveness in preventive care. NDs address the underlying causes of illness or disability by treating the whole person in a way that assists an individual's internal capacity for self-healing. NDs use a variety of treatment modalities, including herbal medicine, nutritional supplementation, physical medicine, homeopathy, lifestyle counseling, and mind-body therapies. Yet, it is their orientation toward holism and vitalism and not specific treatment modalities that unites the profession. Overall, the focus of Naturopathy is health promotion and well being, not simply disease treatment. ${ }^{2}$

Corresponding Author: Mary Koithan, Address: 5861 Placita Roanoke, Tucson, AZ 85704, Phone: (520) 990-6701, FAX: (520) 626-7891, mkoithan@ nursing.arizona.edu.

Publisher's Disclaimer: This is a PDF file of an unedited manuscript that has been accepted for publication. As a service to our customers we are providing this early version of the manuscript. The manuscript will undergo copyediting, typesetting, and review of the resulting proof before it is published in its final citable form. Please note that during the production process errors may be discovered which could affect the content, and all legal disclaimers that apply to the journal pertain. 


\section{Naturopathic Treatment of Obesity}

Naturopathy considers obesity to be less about diet and more about correcting underlying imbalance through lifestyle change that requires a plan of care that supports long-term sustainable health optimization. Six foundational principles inform this plan and can be used to treat obesity.

\section{Let nature heal}

Our bodies have an innate instinct and capacity toward self-healing. By identifying and removing barriers to self-healing, such as poor diet or low self-esteem, NDs nurture this process. Patients are empowered by realizing that they are the source of healing and that external intervention is only there for support.

\section{Identify and treat causes}

Symptoms return unless the root illness is addressed. Rather than suppressing symptoms, NDs identify and treat their root cause. Causes of obesity are complex and multi-factorial, including factors such as lack of awareness, emotional triggers, hormone imbalance, stress, neurotransmitter imbalance, and toxicity. Therefore, treatment plans must be personalized and guidance individualized. In naturopathic medicine, health is the goal and healthy weight is an effect of being healthy. Treatment focuses on attaining overall health (e.g., regaining insulin sensitivity and reversing insulin resistance; optimal digestion; psychological well-being; decreasing stress) not on "weight loss".

\section{First, do no harm}

Naturopathic physicians use low-risk procedures and healing compounds, such as dietary supplements, herbal extracts and homeopathy, with few or no side effects. For example, serotonin 3 and the catecholamines ${ }^{4}$ (dopamine and norepinephrine) are important in the regulation of appetite and satiety. Rather than using medications such as SSRIs and phentermine, an ND might supplement a patient with amino acid precursors (5HTP for serotonin, L-tyrosine and Mucuna pruriens for dopamine and norepinephrine synthesis), thus allowing the body to make the amount of neurotransmitter it needs.

\section{Educate patients}

A primary intervention is patient education. Teaching patients how to eat, exercise and relax/ nurture themselves physically and emotionally is crucial to treatment. A naturopathic doctor might teach a patient about the "glycemic index" or "glycemic load" of foods to guide dietary choices. Alternatively, the ND may recommend the use of yoga for relaxation.

\section{Treat the whole person}

Each individual has a unique physical, mental, emotional, genetic, environmental, social, sexual and spiritual makeup. The ND believes that all these factors contribute to health. In other words, obesity treatment is not likely to succeed unless at least psychosocial, endocrine/ metabolic, and neurochemical aspects of the person are all addressed.

\section{Prevent illness}

The primary focus of naturopathic practice is to promote health and well being, not simply to treat disease. Obesity is one of the most serious public health problems of the $21^{\text {st }}$ century even though it is one of the leading causes of preventable death. NDs evaluate risk factors, heredity and vulnerability to disease. Their practice facilitates and supports lifelong lifestyle change. By addressing greater wellness, it is possible that patients will be less likely to need treatment for future illness. 


\section{Conclusion}

Naturopathic practice is oriented to facilitate patient self-healing and use the clinical encounter as an opportunity to inspire and guide patients to develop a healthier lifestyle. Treatment of obesity is a frequent focus of ND practice. While single therapeutic agents that are used by NDs have undergone testing in research trials, to date there has been no whole-system study that supports the effectiveness of naturopathic medicine for obesity. Yet, the naturopathic philosophy of the healing, including the focus on treating the whole person and preventative care suggest that it is ideally suited for the treatment and prevention of obesity.

\section{Acknowledgments}

This publication was made possible by grants from the National Center for Complementary and Alternative Medicine (NCCAM) [T32 AT001287 and R13 AT005189-01]. Its contents are solely the responsibility of the authors and do not necessarily represent the official views of the NCCAM, or the National Institutes of Health.

\section{References}

1. NIH National Center for Complementary and Alternative Medicine. CAM Basics. [Accessed 6/1/09]. Publication 236. Available at: http://nccam.nih.gov/health/backgrounds/D236.pdf

2. Pizzorno, J.; Murray, M. Textbook of natural medicine. 3. London: Churchill Livingstone Publishers; 2006.

3. Volkow ND, Wang GJ, Maynard L, et al. Brain dopamine is associated with eating behaviors in humans. Int J Eat Disord 2003;33(2):136-42. [PubMed: 12616579]

4. Halford JC, Blundell JE. Pharmacology of appetite suppression. Prog Drug Res 2000;54:25-58. [PubMed: 10857385] 